\title{
Dirofilaria immitis and Angiostrongylus vasorum: the contemporaneous detection in kennels
}

\author{
Luisa Del Prete ${ }^{1}$, Maria Paola Maurelli ${ }^{1}$, Saverio Pennacchio ${ }^{1}$, Antonio Bosco ${ }^{1}$, Vincenzo Musella ${ }^{2}$, Lavinia Ciuca $^{3}$, \\ Giuseppe Cringoli ${ }^{1}$ and Laura Rinaldi ${ }^{1 *}$
}

\begin{abstract}
Background: The cardiopulmonary nematodes Dirofilaria immitis and Angiostrongylus vasorum are increasingly reported in dogs and are responsible for two diseases with overlapping endemic areas, especially in Europe: dirofilariosis and angiostrongylosis. The reasons for their apparent emergence are unknown, but several factors (e.g. increased disease awareness, better diagnostic tools, climatic changes, seasonal population dynamics and movements of animals) may play a role in the recent rise in reports of infection in the various countries of Europe. The aim of this study was to investigate the seroprevalence of D. immitis (by DiroCHECK ${ }^{\circledR}$ ELISA) and the fecal presence of first stage larvae (L1) of A. vasorum (by FLOTAC) in dogs from 68 kennels of the Campania region (southern Italy). The fecal samples were collected from pooled samples using the box as epidemiological unit. To the authors's knowledge, this is the first cross-sectional survey conducted at regional-scale in Italy and in Europe on the contemporaneous detection of D. immitis antigens and A. vasorum L1 in kennels.
\end{abstract}

Results: Antigens of D. immitis were detected in 24/537 (4.4\%; $95 \%$ Confidence Interval = 3.0-6.7) dogs in 6 out of the 68 kennels ( $8.8 \% ; 95 \% \mathrm{Cl}=3.6-18.9)$. The 24 positive samples for D. immitis antigen were tested also with AngioDetect ${ }^{\oplus}$ and only 1 sample was seropositive for $A$. vasorum with a prevalence of $4.2 \%$. A. vasorum L1 were detected in dogs from 9 out of the 68 kennels $(13.2 \% ; 95 \% \mathrm{Cl}=21.8-44.9)$. Pooled fecal samples from 25 boxes out of the 1360 analyzed resulted positive to A. vasorum L1 (1.8\%; $95 \% \mathrm{Cl}=1.2-2.7)$.

Conclusions: The present study indicates that cardiopulmonary nematodes are present in Campania region in symptomatic dogs as well as in asymptomatic ones. Therefore, regular parasitological surveillance, appropriate treatment strategies and high quality standard of hygiene are required to guarantee the health and welfare of kennel dogs.

Keywords: Dirofilaria immitis, Angiostrongylus vasorum, Kennel, FLOTAC, DiroCHEK® ELISA

\section{Background}

The cardiopulmonary nematodes Dirofilaria immitis and Angiostrongylus vasorum are increasingly reported in dogs and are responsible for two diseases with overlapping endemic areas, especially in Europe: angiostrongylosis and dirofilariosis [1-3]. The reasons for their apparent emergence have been discussed in several recent studies; increased disease awareness, the availability

\footnotetext{
* Correspondence: Irinaldi@unina.it

'Department of Veterinary Medicine and Animal Productions, University of Naples Federico II, CREMOPAR Campania Region, Via Della Veterinaria 1, 80137 Naples, Italy

Full list of author information is available at the end of the article
}

of better diagnostic tools and climatic changes are considered the main causes of the recent spread of these parasites [2-7]. In particular, global warming represents a fundamental factor for the seasonality and the spread of $D$. immitis $[7,8]$ and could be involved also in the development of infectious stages in the intermediate hosts of $A$. vasorum $[9,10]$.

The adult stages of $D$. immitis and A. vasorum are both localized in the Arteria pulmonalis and the right heart of their definitive hosts [11]. D. immitis and A. vasorum cause different respiratory signs and severity may change in accordance to the parasite burden and 
host-related factors [12-14]. Age, immune response and presence of concomitant diseases can also determine fatal consequences in the infected animals [13, 14].

Current epidemiological data confirm the expanding trend of $D$. immitis in Italy, as observed in the rest of Europe $[3,15]$. Today $D$. immitis is present not only in the hyperendemic area of the Po River Valley [3, 16], but from northern to southern regions of Italian peninsula as reviewed in Otranto et al. [17], including islands [18] where the infection is now endemic.

Similarly, A. vasorum is undoubtedly spreading from northern to southern Italy, especially in the central regions that offer ideal environmental and epidemiological conditions for the expansion of this parasite and the establishment of further new endemic foci $[19,20]$. Knowledge of the epidemiology and clinical importance of $A$. vasorum has grown in the last 20 years and is correlated especially with increased urbanization of the red fox (Vulpes vulpes) which acts as a reservoir host representing a potential infection for dogs $[21,22]$.

In recent years, epidemiological surveys on $D$. immitis and $A$. vasorum prevalence have been conducted in many countries; however, no studies investigated the simultaneous detection of these cardiopulmonary nematodes in kenneled dogs. Therefore, the aim of this study was to investigate the distribution of $D$. immitis and $A$. vasorum in kenneled dogs of the Campania region of southern Italy. Noteworthy, to the authors' knowledge, this is the first cross-sectional survey conducted at regional-scale in Italy and in Europe on the contemporaneous detection of $D$. immitis antigens and $A$. vasorum first stage larvae (L1) in kennels.

\section{Methods}

\section{Study area and study kennels}

The survey was conducted between June 2012 and August 2013 in the 68 public kennels located in the Campania region of southern Italy (www.anagrafecaninacampania.it). The region (Latitude $=39^{\circ} 59^{\prime} 15^{\prime \prime}-41^{\circ} 30^{\prime} 25^{\prime \prime}$; Longitude $=13^{\circ} 45^{\prime} 25^{\prime \prime}-15^{\circ} 48^{\prime} 23^{\prime \prime}$ ) which extends over an area of $13,590 \mathrm{~km}^{2}$ is mainly hilly and extends from 0 to $1890 \mathrm{~m}$ above sea level. The climate is Mediterranean with dry summers and rainy winters.

As in other Italian regions, the kennels of the Campania region are facilities for roaming and abandoned animals; dogs are sheltered and then given in adoption, if possible. Indeed, the Italian regulation for dog registration/identification (Law 281/1991) provides for setting up specific kennels to collect and re-home roaming and abandoned animals; for many disowned dogs, the kennel becomes a permanent shelter [23]. These kennels are subjected to veterinary public service control. Usually, few anthelmintic treatments per season are used in these kennels, with the number of treatments given per year ranging from 1 (15\%), 2 (75\%) to 3 (10\%) and using broad-spectrum antiparasitic drugs [24]. Routine prophylaxis for $D$. immitis is not performed in the region.

\section{Blood sampling and analysis for D. immitis and A. vasorum}

In the 68 kennels, 537 blood samples (from 5 to 10 per each kennel) were collected. In each kennel we selected the dogs that were hosted for 2 years at least. The samples were transported to the laboratory and centrifuged at $3000 \mathrm{rpm}$ for $10 \mathrm{~min}$ to obtain sera, then stored at $-20{ }^{\circ} \mathrm{C}$ until testing for seroprevalence of D.immitis using DiroCHEK ${ }^{\circ}$ ELISA (Synbiotics, San Diego, USA) according to the manufacturer's instructions (sensitivity $=85$ $100 \%$ and specificity $=100 \%)[25]$.

After the analysis with DiroCHEK ${ }^{\circ}$ ELISA, the dog sera resulted positive to $D$. immitis were also tested with the antigenic test AngioDetect ${ }^{\circ}$ (IDEXX Laboratories, Westbrook, Maine, USA) (sensitivity $=84.6 \%$; specificity $=100 \%$ ) [26]. The blood samples were collected by the permission of the dog shelters. The animals used in the present study were sampled following approval by the animal ethics and welfare committee of the University of Naples Federico II (protocol number 0093412).

\section{Fecal sampling and analysis for A. vasorum}

In each of the 68 studied kennels, the box (hosting a different number of dogs, i.e. $\min 1, \max 4$; mean $=2.4$ dogs) was considered as the epidemiological unit of the study for practical reasons as reported in Rinaldi et al. [24]. In each kennel, 20 boxes were sampled by systematic sampling (total number of boxes $=1360$ ). In each box, the fecal samples were collected directly from the ground (pooled samples), placed in a container and transported to the laboratory (within $5 \mathrm{~h}$ from sampling). The feces were fixed in $5 \%$ formalin (dilution ratio 1:4). Copromicroscopic analyses were performed using the FLOTAC technique, having an analytic sensitivity of 2 larvae per gram (LPG) of feces. A zinc sulphate-based solution (specific gravity $=1.20$ ) was used for diagnosis of A. vasorum [27].

\section{Results and discussion}

Antigens of $D$. immitis were detected in dogs from 6 out of the 68 kennels $(8.8 \%$; $95 \% \mathrm{CI}=3.6-18.9)$. Specifically, 24/537 (4.4\%; $95 \% \mathrm{CI}=3.0-6.7)$ dogs of 6 kennels were positive to $D$. immitis (Table 1 ). The 24 sera resulted positive to $D$. immitis were tested also with AngioDetect ${ }^{\oplus}$ in order to detect possible cross-reactions [11]. Only 1 sample ( $4.2 \% ; 95 \% \mathrm{CI}=0.2-23.1)$ resulted positive for $A$. vasorum antigens. This dog did not show any clinical sign and $A$. vasorum L1 were detected in the corresponding box (see below). 
Table 1 Prevalence of Dirofilaria immitis antigen at individual level in the positive kennels, $n=6$

\begin{tabular}{lllcc}
\hline Kennel ID & No. of dogs examined & No. of dogs positive to Dirofilaria immitis antigen & Prevalence (\%) & $95 \%$ confidence interval \\
\hline 1 & 20 & 5 & 25.0 & $9.6-49.4$ \\
2 & 93 & 5 & 5.3 & $2.0-12.7$ \\
3 & 46 & 3 & 6.5 & $1.7-18.9$ \\
4 & 94 & 6 & 4.2 & $2.6-13.9$ \\
5 & 94 & 4 & 4.2 & $1.4-11.2$ \\
6 & 9 & 1 & 11.0 & $0.6-49.3$ \\
Total & 356 & 24 & & \\
\hline
\end{tabular}

A. vasorum (L1) were detected in dogs from 9 out of the 68 kennels $(13.2 \%$; $95 \% \mathrm{CI}=21.8-44.9$; LPG $\mathrm{min}=$ 4; $\mathrm{LPG} \max =106$; LPG mean $=25.1$ ). Pooled fecal samples from 25 boxes out of the 1360 analyzed resulted positive to $A$. vasorum L1 (1.8\%; $95 \% \mathrm{CI}=1.2-2.7)$. The mean number of $A$. vasorum positive boxes/kennel was $2.8(\min 1-\max 7)$.

The strength of the present study was the contemporaneous detection of $D$. immitis antigens (8.8\%) and $A$. vasorum L1 (13.2 \%) in kennels conducted by a crosssectional survey at regional-scale.

However, also some limits emerged from our study. First, the lack of knowledge of the history of the kenneled dogs; we did not know if they were all natives of the Campania Region, even if autochthonous foci of canine $D$. immitis infection were recently reported in owned dogs from the same region [28]. Second, the impossibility to collect the blood from all the dogs examined by coprological techniques due to the aggressiveness of the kennel animals. Third, the using of pooled fecal samples; the percentage of positivity for A. vasorum found in this study (13.2\%) cannot directly be compared with prevalence of other study based on individual samples.

The main findings of the present survey confirm that polyparasitism is the rule in the studied kennels in southern Italy as already described by Rinaldi et al. [24] concerning other endoparasites. Different considerations emerged from this study related to: (i) epidemiology and (ii) diagnosis and control of cardiopulmonary nematodes infecting dogs.

D. immitis has spread in the last years in Europe due to climate change, the spreading of mosquito Aedes albopictus and the introduction of new vector Aedes koreicus [29]. These factors caused new autochthonous foci of heartworm disease that have recently been reported in previously non-endemic areas of southern Italy [8, 30]. While the distribution and prevalence of $D$. immitis infection has been widely studied in dogs from central and northern Italy, epidemiological data on the occurrence of $D$. immitis infection in southern Italy are scant and limited to sporadic case reports. The prevalence of D. immitis reported in this study was $8.8 \%$. This prevalence is higher than the previous study conducted in the Vesuvius area $(0.6 \%)$ of the same region confirming the spread of this parasite [28]. Similarly, also in Apulia and Calabria regions D. immitis was found in autochthonous dogs [17].

Different predictive studies, conducted using Geographical Information Systems (GIS) tools [15, 16], confirmed a correlation between the actual trend of $D$. immitis spread and the temperature increase into previously infection free areas as demonstrated also in a questionnaire survey by Genchi et al., [31] where an increasing number of positive cases to $D$. immitis was detected in non- endemic area (10\%) and in endemicarea (12\%) of Europe.

A. vasorum infection is considered endemic in certain areas of Europe, including regions of Denmark, Germany, Hungary, Finland, France, Ireland, Italy, the Netherlands, Poland, Slovakia, Spain, Sweden, Switzerland, Turkey and the United Kingdom. The country list is growing as the $A$. vasorum geographic distribution continues to evolve in several countries of Europe [13].

Our present study expands on that recently published by Rinaldi et al [32] which described a case of fatal disseminated $A$. vasorum infection in the same region. Also in the same region a prevalence of $33.3 \%$ was found in red foxes at post-mortem examination [21] and the authors indicated the potential infection risk for dogs living in the same area. The most important and best-studied wildlife reservoir for $A$. vasorum infection is the red fox (Vulpes vulpes) [13, 22]. A possible explanation for increased transmission of infection between red fox and dog populations is an increasing density of foxes. It can be assumed that an increased density of foxes in an area populated with dogs is likely to increase the number of fox-dog interactions, and hence increase the opportunity for transmission of infection. Direct wild canid-dog interaction is not necessary for (and does not lead to) transmission of this parasite from one to the other because transmission occurs via ingestion of L3 (in gastropods or frogs, or possibly free in the environment) [13].

The positive percentage found in our present study (13.2\%), even if is not comparable with the prevalence 
of the other study because of the different epidemiological unit considered, is very high if compared other studies [33, 34]. The scenario of A. vasorum infection is alarming if we consider the clinical signs can be severe: respiratory dysfunctions, bleeding, neurological, ocular, cardiovascular and gastrointestinal symptomatology, skin lesions [12, 33]. Even if current data of this disease are referred to symptomatic dogs, A. vasorum infection may be also asymptomatic, especially in the early stages and so affected dogs can develop clinical signs after months to years from the infection [13].

Kennel dogs are at the highest risk of infection with helminths, for this reason anthelmintics or combinations of anthelmintics with a broad spectrum activity, as suggested in a previous study [24], are required to treat the polyparasitism currently encountered in this situations. Unfortunately, the anthelmintics used still now do not work as preventives of $D$. immitis. The reasons of the lack of a prophylaxis for $D$. immitis are: the unawareness of the severity of this parasite, the economic issue and the difficulties of managing and monitoring of the kenneled dogs.

\section{Conclusion}

In conclusion, the present study indicates that the cardiopulmonary nematodes $D$. immitis and $A$. vasorum circulate in the kennels of the Campania region of southern Italy. Therefore, regular parasitological surveillance, appropriate diagnostic tools, treatment strategies and high quality standard of hygiene are required to guarantee the health and welfare of kennel dogs as recommended by the European Scientific Counsil for Companion Animals Parasites (www.esccap.org).

\section{Abbreviations}

Cl: confidence interval; L1: first stage larvae; LPG: Larvae per gram.

\section{Competing interests}

The authors declare that they have no competing interests.

\section{Authors' contributions \\ LDP - Carried out the laboratory analysis, the interpretation of data and drafted the manuscript. MPM - Carried out the laboratory analysis, the interpretation of data and contributed to the writing of the manuscript. SP and $\mathrm{AB}$ - Collected the samples and carried out the laboratory analysis. VM- Carried out the interpretation of data. LC-Carried out the laboratory analysis and the interpretation of data. GC-Conceived the study and revised the manuscript. LR- Conceived the study, participated in its design and coordination, performed the statistical analysis and revised the manuscript. All authors read and approved the final version of the manuscript.}

\section{Acknowledgements}

The work was conducted under the frame of EurNegVec COST Action TD1303.

\section{Author details}

'Department of Veterinary Medicine and Animal Productions, University of Naples Federico II, CREMOPAR Campania Region, Via Della Veterinaria 1, 80137 Naples, Italy. ${ }^{2}$ Department of Health Sciences, University Magna Graecia of Catanzaro, Catanzaro, Italy. ${ }^{3}$ Jon lonescu de la Brad", University of Agricultural Sciences and Veterinary Medicine, lasi, Romania.
Received: 6 August 2015 Accepted: 16 December 2015

Published online: 21 December 2015

\section{References}

1. Maia C, Coimbra M, Ramos C, Cristovao JM, Cardoso L, Campino L. Serological investigation of Leishmania infantum, Dirofilaria immitis and Angiostrongylus vasorum in dogs from southern Portugal. Parasit Vectors. 2015;8:152.

2. Morgan ER, Jefferies R, van Otterdijk L, McEniry RB, Allen F, Bakewell M, et al. Angiostrongylus vasorum infection in dogs: presentation and risk factors. Vet Parasitol. 2010:173(3-4):255-2561.

3. Otranto D, Dantas-Torres F, Brianti E, Traversa D, Petric D, Genchi C, et al. Vector-borne helminths of dogs and humans in Europe. Parasit Vectors. 2013;6:16

4. Morgan ER. Canine pulmonary angiostrongylosis: can a worm change its spots? Vet Rec. 2014;175(5):116-7.

5. Tolnai Z, Széll Z, Sréter T. Environmental determinants of the spatial distribution of Angiostrongylus vasorum, Crenosoma vulpis and Eucoleus aerophilus in Hungary. Vet Parasitol. 2015;207(3-4):355-8.

6. Helm J, Roberts L, Jefferies R, Shaw SE, Morgan ER. Epidemiological survey of Angiostrongylus vasorum in dogs and slugs around a new endemic focus in Scotland. Vet Rec. 2015;177(2):46.

7. Genchi C, Mortarino M, Rinaldi L, Cringoli G, Traldi G, Genchi M. Changing climate and changing vector-borne disease distribution: The example of Dirofilaria in Europe. Vet parasitol. 2011;176(4):295-99.

8. Morchon R, Carrètòn E, Gonzalez-Miguel J, Mellado-Hernandez I. Heartworm disease (Dirofilaria immitis) and their vectors in Europe- New distribution trends. Front Physiol. 2012;3:196

9. Traversa D, Di Cesare A, Conboy G. Canine and feline cardiopulmonary parasitic nematodes in Europe: emerging and underestimated. Parasit Vectors. 2010;23:3-62.

10. Morgan ER, Jefferies R, Krajewski M, Ward P, Shaw SE. Canine pulmonary angiostrongylosis: the influence of climate on parasite distribuition. Parasitol Int. 2009;58(4):406-10.

11. Schnyder M, Deplazes P. Cross-reactions of sera from dogs infected with Angiostrongylus vasorum in commercially available Dirofilaria immitis test kits. Parasit Vectors. 2012;5:258.

12. Koch J, Willesen JL. Canine pulmonary angiostrongylosis: an update. Vet J. 2009:179(3):348-59.

13. Elsheikha HM, Holmes SA, Wright I, Morgan ER, Lacher DW. Recent advances in the epidemiology, clinical and diagnostic features, and control of canine cardio- pulmonary angiostrongylosis. Vet Res. 2014;45:92.

14. McCall JW, Genchi C, Kramer LH, Guerrero J, Venco L. Heartworm disease in animals and humans. Adv Parasitol. 2008;66:193-285.

15. Genchi C, Rinaldi L, Mortarino M, Genchi M, Cringoli G. Climate and Dirofilaria infection in Europe. Vet Parasitol. 2009;26;163(4):286-92.

16. Genchi C, Rinaldi L, Cascone C, Mortarino M, Cringoli G. Is heartworm disease really spreading in Europe? Vet Parasitol. 2005;133(2-3):137-48.

17. Otranto D, Capelli $G$, Genchi C. Changing distribution patterns of canine vector borne diseases in Italy: Leishmaniosis vs. dirofilariosis. Parasit Vectors. 2009;2(Suppl I):S2.

18. Pipia AP, Varcasia A, Tosciri G, Seu S, Manunta ML, Mura MC, et al. New insights onto cardiopulmonary nematodes of dogs in Sardinia, Italy. Parasitol Res. 2014;113(4):1505-9.

19. Di Cesare A, Castagna G, Meloni S, Milillo P, Latrofa S, Otranto D, et al. Canine and feline infections by cardiopulmonary nematodes in central and southern Italy. Parasitol Res. 2011;1:587-96.

20. Guardone L, Schnyder M, Macchioni F, Deplazes P, Magi M. Serological detection of circulating Angiostrongylus vasorum antigen and specific antibodies in dogs from central and northern Italy. Vet Parasitol. 2013;192(1-3): $192-8$.

21. Santoro M, D'Alessio N, Di Prisco F, Neola B, Restucci B, Pagano TB, et al. Angiostrongylus vasorum infection in red foxes (Vulpes vulpes) in southern Italy. Acta Parasitol. 2015;60(2):356-9.

22. Taylor CS, Garcia Gato R, Learmount J, Aziz NA, Montgomery C, Rose H, et al. Increased prevalence and geographic spread of the cardiopulmonary nematode Angiostrongylus vasorum in fox populations in Great Britain. Parasitology. 2015;142(9):1190-5.

23. Baldelli R, Piva S, Salvatore D, Parigi M, Melloni O, Tamba M, et al. Canine leishmaniasis surveillance in a northern Italy kennel. Vet Parasitol. 2011;179:57-61. 
24. Rinaldi L, Pennacchio S, Musella V, Maurelli MP, La Torre F, Cringoli G. Helminth control in kennels: is the combination of milbemycin oxime and praziquantel a right choice? Parasit Vectors. 2015;17:8(1):30.

25. Courtney $\mathrm{CH}$, Zeng Q. Comparison of heartworm antigen test kit performance in dogs having low heartworm burdens. Vet Parasitol. 2001; 317-322.

26. Schnyder M, Stebler K, Naucke TJ, Lorentz S, Deplazes P. Evaluation of a rapid device for serological in-clinic diagnosis of canine angiostrongylosis. Parasit Vectors. 2014;18(7):72

27. Schnyder M, Maurelli MP, Morgoglione ME, Kohler L, Deplazes $P$, Torgerson $\mathrm{P}$, et al. Comparison of faecal techniques including FLOTAC for copromicroscopic detection of first stage larvae of Angiostrongylus vasorum. Parasitol Res. 2011;109(1):63-9.

28. Rinaldi L, Del Prete L, Noviello E, Musella V, Cringoli G. Dirofilaria infection in dogs from the Campania Region of southern Italy. Second Conference on Neglected Vectors and Vector-borne diseases (EurNegVec) with management and working group meetings on the COST ACTION TD1303, Izmir, Turkey, March 31- April 2; 2015.p.80.

29. Montarsi F, Ciocchetta S, Devine G, Ravagnan S, Mutinelli F, Frangipane di Regalbono A, et al. Development of Dirofilaria immitis within the mosquito Aedes (Finlaya) koreicus, a new invasive species for Europe. Parasit Vectors. 2015;8:177.

30. Otranto D, Dantas-Torres F. Canine and feline vector-borne diseases in Italy: current situation and perspectives. Parasit Vectors. 2010;3:2.

31. Genchi C, Bowman D, Drake J. Canine heartworm disease (Dirofilaria immitis) in Western Europe: survey of veterinary awareness and perceptions. Parasit Vectors. 2014;7:2.

32. Rinaldi L, Cortese L, Meomartino L, Pagano TB, Pepe P, Cringoli G, et al. Angiostrongylus vasorum: epidemiological, clinical and histopathological insights. BMC Vet Res. 2014;10:236.

33. Traversa D, Di Cesare A, Meloni S, Frangipane di Regalbono A, Milillo P, Pampurini F, et al. Canine angiostrongylosis in Italy: occurrence of Angiostrongylus vasorum in dogs with compatible clinical pictures. Parasitol Res. 2013;112(7):2473-80

34. Tieri E, Pomilio F, Di Francesco G, Saletti MA, Totaro P, Troilo M, et al. Angiostrongylus vasorum in 20 dogs in the province of Chieti, Italy. Vet Ital. 2011:47(1):77-88.

\section{Submit your next manuscript to BioMed Central and we will help you at every step:}

- We accept pre-submission inquiries

- Our selector tool helps you to find the most relevant journal

- We provide round the clock customer support

- Convenient online submission

- Thorough peer review

- Inclusion in PubMed and all major indexing services

- Maximum visibility for your research

Submit your manuscript at www biomedcentral.com/submit 